\title{
LA IMPRONTA EMOCIONAL EN EL PERFIL NEUROEDUCATIVO DEL DOCENTE UNIVERSITARIO
}

\author{
A impressão emocional no perfil neuroeducacional do profesor universitário \\ Quispe Palomino, Jaime ${ }^{1}$ \\ Universidad Nacional del Centro del Perú
}

\begin{abstract}
Resumen
El estudio tiene como propósito principal establecer rasgos peculiares y distintivos en el perfil del docente universitario, denominada por la neuroeducación impronta emocional (Bain, 2007, Mora, 2021) y promovida como uno de los componentes que motivan el ejercicio de la labor excelente y excepcional del docente universitario; sabiendo que la universidad, escenario de acción del docente universitario, atraviesa tiempo de cambio y transformación, en el siglo del cerebro que, para consolidar profesionalmente su aprendizaje, necesita siempre emocionarse; así, poner en práctica el pensamiento crítico, analítico y creativo.

Esta investigación es de tipo descriptiva con análisis e interpretación basada en la realidad actual. Según el objeto de estudio es una investigación pura. Se utilizó el método descriptivo correlacional y no experimental.

Se hizo un estudio de recopilación de información de libros, artículos y tesis de investigación que abordan el perfil del docente universitario, dentro del complejo sistema educativo, buscando el equilibrio de la calidad educativa que trata no solo de la erudición cognitivo sino también de impronta emocional que enfatiza la neuroeducación. Precisamente, basado en las normas educativas, universitarias, nacionales y mundiales se propone diecisiete características que conforman el perfil neuroeducativo del docente universitario.
\end{abstract}

Palabras clave: Impronta emocional, perfil educativo, docente universitario, neuroeducación, calidad educativa

\section{Resumo}

O objetivo principal do estudo é estabelecer características peculiares e distintivas no perfil do professor universitário, denominadas por neuroeducação de cunho emocional (Bain, 2007, Mora, 2021) e promovidas como um dos componentes que motivam o exercício de excelentes e excepcionais trabalho do professor universitário; sabendo que a universidade, palco de ação do professor universitário, vive um momento de mudança e transformação, no século do cérebro que, para consolidar profissionalmente seu aprendizado, precisa sempre se animar; assim, colocar em prática o pensamento crítico, analítico e criativo. Esta pesquisa é descritiva com análise e interpretação com base na realidade atual. Segundo o objeto de estudo, trata-se de uma investigação pura. Foi utilizado o método descritivo, correlacional e não experimental. Realizou-se um estudo para coletar informações em livros, artigos e teses de pesquisa que abordam o perfil do professor universitário, dentro do complexo sistema educacional, buscando o equilíbrio da qualidade educacional que não é apenas sobre bolsa cognitiva, mas também sobre a impressão emocional que a neuroeducação enfatiza tanto, em Francisco Mora e outros inovadores da educação. Também foram levados em consideração a Lei Universitária Peruana, o Projeto Nacional de Educação até 2036 e as Metas de Desenvolvimento Sustentável até 2030 (objetivo 4).

Precisamente, a partir de padrões educacionais, universitários, nacionais e mundiais, são propostas dezessete características que compõem o perfil neuroeducacional dos professores universitários.

Keywords: impressão emocional, perfil educacional, professor universitário, neuroeducação, quialidade educacional.

\section{Introducción}

El presente siglo XXI está marcado por una experiencia de vida conocida como la "era del cerebro" (D'Addario, 2019, p. 10), impulsada por la neurociencia, no como una moda sino una necesidad. Cada vez más crece el

\footnotetext{
${ }^{1}$ Correspondencia: jaime.quispe0015@gmail.com, https://orcid.org/0000-0002-4534-5083
} 
interés por estudiar el desarrollo, estructura y funcionamiento del sistema nervioso en el hombre; y se consolida transdisciplinariamente con las ciencias sociales y humanas, como la educación, psicología, sociología, medicina, filosofía, ética, etc. buscan desarrollar una concepción integradora.

La neuroeducación es pues una ciencia emergente que articula la aplicación de los conocimientos, dentro proceso de enseñanza-aprendizaje, en el funcionamiento del cerebro del estudiante por "un intento de mejorar y potenciar tanto los procesos de aprendizaje y memoria de los estudiantes como enseñar mejor en los profesores" (Mora, 2021, p.222); teniendo en cuenta el binomio consubstancial e indisoluble "emocióncognición" (Mora, 2021, p. 69). Es necesario tener en cuenta las diversas denominaciones que está adquiriendo la neuroeducación, o al menos las diversas perspectivas con las que se está abordando, tales como neurociencia cognitiva, neurociencia aplicada a la educación, neuropsicología, neurodidáctica, etc.; así como los diversos componentes que la integran y se deben tener en cuenta, tal es el caso de la impronta emocional que se abordará en la presente investigación.

Si la neuroeducación, como dice Mora, intenta mejorar los procesos de aprendizaje o mentales en el binomio "emoción-cognición" (Mora, 2021, p. 71), tanto en el estudiante como en el docente, la presente investigación establecerá la impronta emocional del docente universitario, entendido como rasgos peculiares y distintivos, que motive la excelencia y excepcionalidad de su profesión en el proceso de enseñanza-aprendizaje de los estudiantes que serán los protagonistas de un mundo mejor.

Hablar de la "impronta emocional" es poner en primer plano la importancia que tienen las emociones, como punto de partida o "energía que mueve el mundo" (Mora, 2021, p. 69), dentro del proceso educativo de enseñanza-aprendizaje; por tratarse de "un ingrediente básico del proceso cognitivo, del razonamiento" (Mora, 2021, p. 71).

Por tanto, la presente investigación planteará diecisiete características neuroeducativas que se deben promover en el campus universitario con la finalidad de hacer de los docentes "profesores universitarios excelentes" (Mora, 2021, p. 196) que destaquen los rasgos peculiares y distintivos, expresados en la Ley Universitaria 30220 (2014), el Sistema Nacional de Evaluación, Acreditación y Certificación de la calidad (DS N018-2007-ED), el Proyecto Educativo Nacional al 2036 (2020) y Los Objetivos de Desarrollo Sostenible al 2030 (2015).

\section{Perfil neuroeducativo del docente universitario}

Resulta complejo hablar del perfil neuroeducativo del docente universitario en plena disrupción de la educación porque, a nivel mundial, existen diversas concepciones de la educación superior, en pleno tiempo de cambio, a la cual está llamado responder eficientemente a los problemas que atraviesa la humanidad.

Ante esta situación emergente surgen exigencias ipso facto, por parte de los docentes, para responder con inmediatez y versatilidad, la impronta emocional ante los crecientes cambios de paradigmas neuroeducativos ya que "la universidad, para aquellos que han tenido la posibilidad de acceder a ella, es donde el cerebro continúa formándose y transformándose de un modo clave y determinante... en su vida futura tanto profesional como personal" (Mora, 2021, p. 192).

Moreno y Marcaccio (2014), en la búsqueda de una definición, sostienen que "el término de perfil profesional suele hacer referencia tanto al producto de una experiencia educativa como a las características que posee un sujeto. Hasta el momento, los diferentes autores no han arribado a una única definición de perfil profesional" (p. 130). Es por eso que se tendrán una definitiva reorganización, mucho más ahora, ante la situación disruptiva de cambios determinantes, caminos para una sólida definición.

Ocampo et al (2014, como se citó en del Águila, 2019) dice, de manera específica, que:

el docente universitario en aquél que acredita conocimientos en el área que acompaña, experiencia investigativa y profesional en las áreas de desempeño; y, que identifica necesidades y acciones formativas, ... en el marco de un programa general y guía a los estudiantes hacia el logro de los objetivos de aprendizaje (p. 22)

Es por eso que el docente debe tener en cuenta diversas herramientas y estrategias didácticas, en el proceso de enseñanza-aprendizaje, porque "también es importante tener en cuenta otro tipo de competencias, denominadas transversales (dado que se desarrollan durante todo el grado) como las competencias básicas, las tecnológicas y las comunicativas" (p. 4)

Además, se debe tener en cuenta, en el plano actual del pensamiento complejo, la multidisciplinariedad en la era del conocimiento. Por lo que se debe definir un perfil de este "constructor de voluntades" representa una tarea compleja, que pudiera tener sus creadores en teorías y modelos que explican desde indicadores y rasgos 
basados en conocimientos y disciplinas abstractas hasta llegar a concebir el perfil basado en competencias tal como se asume en el proyecto Tuning (Oviedo, 2003, párr. 3).

Tal es así que los estudiantes que entran en las aulas universitarias tienen competencias heterogéneas como la excelente preparación cognitiva, habilidades múltiples, contextos emocionales variados, expectativas profesionales, actitudes críticas y creativas.

Por tanto, el perfil del docente consiste en interpretar los signos de los tiempos y actuar de acuerdo a las circunstancias y necesidades que experimenta la sociedad actual, acompañando el proceso educativo complejo de manera cognitiva y emocional. En efecto, la neuroeducación proporciona, en el proceso investigativo realizado, científicamente por la neurociencia, estudios que mejoren la calidad de vida en el estudiante que "ayude a crecer en dignidad" (Bueno, 2021, p.16). Si se pretende calidad de vida en el estudiante, el docente debe contar con las herramientas suficientes para poder hacerlo. Si se pretende un perfil de calidad para el docente, la institución debe tener condiciones básicas de calidad humana, basados en el sostenimiento emocional que actualmente promueve la neuroeducación. Si las instituciones educativas velan por el estado emocional de los docentes, el mundo será mejor y feliz. De allí que, entre las herramientas proporcionadas por la neurociencia aplicada a la educación, se encuentran, dentro del perfil del docente universitario, las características, previamente concientizadas, en el cerebro del docente universitario y puestas en movimiento a través del circuito de redes neuronales (Mora, 2021, p. 20), generada por la impronta emocional que mueve al docente de su acostumbrada y tradicional zona de confort de hacer siempre lo mismo y transformar, gracias a los rasgos peculiares y distintivos, en docente excepcional y excelente que dejen huellas en los estudiantes.

\section{La Ley Universitaria}

La Constitución Política del Perú (1993), dice que "la educación universitaria tiene como fines la formación profesional, la difusión cultural, la creación intelectual y artística y la investigación científica y tecnológica" (artículo 18). A partir de estas categorías mencionadas por la carta magna se construirá el perfil neuroeducativo del docente universitario que responda la confluencia armónica del bien supremo que vela el estado, que vive la sociedad, que enseña el docente, que espera la familia y pone en práctica el estudiante.

La Ley Universitaria peruana 30220 (2014) "promueve el mejoramiento continuo de la calidad educativa de las instituciones universitarias como entes fundamentales de desarrollo nacional, de la investigación y de la cultura" (artículo 1). Se destaca el objetivo fundamental de regular la "calidad educativa" que promuevan el desarrollo, la investigación y la cultura en nuestro país.

El Capítulo VIII aborda todo lo relacionado al docente en el ejercicio de sus funciones como "la investigación, el mejoramiento continuo y permanente de la enseñanza, la proyección social y la gestión universitaria" (artículo 79). Definitivamente, estas funciones se convierten en orientaciones que indican los caminos que debe recorrer el docente, como acompañante del estudiante que debe conseguir un grado de reconocimiento emocional y cognitivo. "Reconocimiento que no solo se refiere a las enseñanzas expresadas en sus clases magistrales, sino en ser personas capaces de ser guía en la formación de las nuevas generaciones" (Mora, 2021, p. 191) que tienen que afrontar retos y desafíos sostenibles en educación, salud, alimentación, libertad, justicia, inclusión, igualdad, economía, ecología, medio ambiente, etc. con el propósito de alcanzar vivir en una ciudadanía plena donde los jóvenes con buen estado de salud física y mental que desarrollen aprendizajes necesarios para desenvolverse con solvencia en el rubro de su preferencia, que ejerzan plenamente su ciudadanía y contribuyan a construir una sociedad democrática y sostenible desde sus proyectos personales y colectivos (Proyecto Educativo Nacional al 2036, 2020, p. 35).

Los deberes (artículo 87) y derechos (artículo 88) del docente universitario están planteados desde los principios (artículo 5), fines (artículo 6) y funciones (artículo 7) de la universidad que se citarán a continuación. 


\section{Figura 1 Principios de la Ley Universitaria}

\section{Artículo 5. Principios}

Las universidades se rigen por los siguientes principios:

5.1 Búsqueda y difusión de la verdad.

5.2 Calidad académica.

5.3 Autonomía.

5.4 Libertad de cátedra.

5.5 Espíritu crítico y de investigación.

5.6 Democracia institucional.

5.7 Meritocracia.

5.8 Pluralismo, tolerancia, diálogo intercultural e inclusión.

5.9 Pertinencia y compromiso con el desarrollo del país.

5.10 Afirmación de la vida y la dignidad humana.

5.11 Mejoramiento continuo de la calidad académica.

5.12 Creatividad e innovación.

5.13 Internacionalización.

$5.14 \mathrm{El}$ interés superior del estudiante.

\section{Fuente: Ley Universitaria $N^{\circ} 30220$}

\section{Figura 2: Fines de la universidad}

\section{Artículo 6. Fines de la universidad}

La universidad tiene los siguientes fines:

6.1 Preservar, acrecentar y transmitir de modo permanente la herencia científica, tecnológica, cultural y artística de la humanidad.

6.2 Formar profesionales de alta calidad de manera integral y con pleno sentido de responsabilidad social de acuerdo a las necesidades del país.

6.3 Proyectar a la comunidad sus acciones y servicios para promover su cambio y desarrollo.

6.4 Colaborar de modo eficaz en la afirmación de la democracia, el estado de derecho y la inclusión social.

6.5 Realizar y promover la investigación científica, tecnológica y humanística la creación intelectual y artística.

6.6 Difundir el conocimiento universal en beneficio de la humanidad.

6.7 Afirmar y transmitir las diversas identidades culturales del país.

6.8 Promover el desarrollo humano y sostenible en el ámbito local, regional, nacional y mundial.

6.9 Servir a la comunidad y al desarrollo integral.

6.10 Formar personas libres en una sociedad libre.

\section{Fuente: Ley Universitaria $\mathrm{N}^{\circ} \mathbf{3 0 2 2 0}$}

Figura 3: Funciones de la universidad

\section{Artículo 7. Funciones de la universidad}

Son funciones de la universidad:

7.1 Formación profesional.

7.2 Investigación.

7.3 Extensión cultural y proyección social.

7.4 Educación continua.

7.5 Contribuir al desarrollo humano.

7.6 Las demás que le señala la Constitución Política del Perú, la ley, su estatuto y normas conexas.

Fuente: Ley Universitaria $N^{\circ} 30220$

Dichos principios, fines y funciones se convierten en pilares que unirán esfuerzos en lograr el propósito de la "calidad educativa" que rigen el modelo de universidad como institución, el profesionalismo de los docentes y, sobre todo, el beneficio que obtienen los estudiantes y graduados (artículo 3) con el único sueño de "que el Perú pueda tener un proyecto educativo donde todas las personas, a lo largo de su vida, puedan estudiar, ser mejores peruanos, ser mejores personas, ser mejores profesionales" (PEN al 2036, 2020, p. 40) mediante sus cuatro 
propósitos; primero, vida ciudadana; segundo, inclusión y equidad; tercero, bienestar socioemocional; cuarto, prosperidad, productividad, investigación y sostenibilidad.

Dichas normas son pues el fundamento y plataforma del perfil profesional del neuroeducador universitario que desbarata la tradicional educación vertical, pensada solamente en la erudición cognitiva del estudiante e integrar la necesaria impronta emocional del estudiante que le hace consciente de vivir, protagonista de su propia transformación y ser constructores de una ciudadanía plena porque, como dice Mora (2021), "la universidad, esa 'unidad' que vertebra el conocimiento, es una institución de trascendencia para otear en el horizonte los cambios culturales que se avecinan y en donde lo humano, la enseñanza personal, directa, del 'maestro' seguirá siendo fundamental" (p. 191) y como estipula el objetivo de la Ley Universitaria trabajar juntos en el "aseguramiento de la calidad de la educación superior universitaria" (artículo 1).

\section{La impronta emocional del docente universitario}

Mora (2021), citó un experimento hecho por tres investigadores universitarios, en la década de los setenta, para medir el componente emocional de sus estudiantes al momento de realizar el proceso de enseñanza-aprendizaje (p. 195; Bain, 2007, p. 8). El experimento consistió en contratar un actor para dar las clases. Para eso, se debían tener en cuenta dos consignas principales; primero, que las clases estuvieran cargadas de alto contenido emocional que les divirtiera de la mejor manera posible; segundo, que el contenido que se tenía que dar sea deficiente y confuso. Después de un determinado tiempo se pidió a los estudiantes que calificaran y los resultados fueron muy buenos. A esto le llamaron "efecto Dr. Fox" (Bain, 2007, p. 15).

Cabe la siguiente pregunta: ¿Qué hizo que los estudiantes universitarios, después de un período de clases, sintieran satisfacción, a pesar de que no se les estaba dando un aprendizaje satisfactorio que les sirviera para la vida profesional de los estudiantes?

Compleja realidad al momento de analizar. Las respuestas serán todavía más complejas de lo que se podría pensar. Cabe aclarar, desde un principio, que el presente artículo no tiene el propósito de dar soluciones ni buscar culpables. Se trata más bien de reflexionar críticamente el pensamiento complejo, a la luz de la realidad educativa. Para ello se debe tener en cuenta las fortalezas y debilidades, aciertos y desaciertos, riesgos y oportunidades, vigencia de modelos educativos y acontecimientos históricos. Al respecto, Edgar Morin (2011) dice, pues, que "no se trata de retomar la ambición del pensamiento simple de controlar y dominar lo real. Se trata de ejercitarse en un pensamiento capaz de tratar, de dialogar, de negociar, con lo real" (p. 10).

Es así que el perfil neuroeducativo del docente universitario no consiste en comparar, descartar, entrar en la moda y dejarse envolver por un vendaval de novedades, sino de tomar conciencia de sus rasgos peculiares y distintivos que impriman emocionalmente características esenciales que conduzcan al ejercicio de su profesión pero de manera excelente y excepcional (Mora, 2021, pp. 192-195) para celebrar y vivir intensamente el proceso educativo del presente que no llegó improvisadamente sino para dar respuestas a los signos de los tiempos y de proyectarse a vivir un mundo mejor donde haya calidad, equidad, inclusión y sostenibilidad educativa. Se trata de hablar de una educación para forjar una ciudadanía plena (PEN al 2036, 2020, pp. 13-17) donde el estudiante aprende lo que ama, lo que le motiva, lo que le emociona, lo que le hace libre y lo que le dignifica. Definitivamente, el cerebro necesita encontrar el disfrute por el placer de aprender.

Hoy en día se acepta más la necesidad e importancia que tienen la unión y reunión de las ciencias para buscar una solución consensuada a los problemas educativos relacionados a la realidad social, ambiental, económica, sanitaria, ética, etc. Sobre todo, demostrar el mutuo enriquecimiento de los aportes que tengan que hacer las disciplinas en la búsqueda del bien común de la educación, unidos consustancialmente en los progresos significativos de la historia de la humanidad. De esta manera, el servicio educativo, por ley de gravedad, tendrá que ser transdisciplinar, multidisciplinar, interdisciplinar y pluridisciplinar (Fuentes y Collado, 2019, pp. 87-91). Al experimento, citado por Mora, intervinieron varias disciplinas que tuvieron que erradicar los neuromitos, instalados dentro del sistema educativo. La neuroeducación, para responder a la situación, recurrió a los experimentos y estudios de la neurociencia, basada en el "desarrollo, estructura, función, farmacología y patología del sistema nervioso" (Mora, 2021, p.221). También se integró "con la psicología, la sociología y la medicina en un intento de mejorar y potenciar tanto los procesos de aprendizaje y memoria de los estudiantes como enseñar mejor en los profesores" (Mora, 2021, p. 29). Por su naturaleza reflexiva, está la filosofía que argumenta el origen, las causas, el sentido y significado de los problemas educativos, personal e integralmente; junto a él, también, se encuentra la ética, basada en "los valores [que] tienen como sustrato neurobiológico principal los circuitos y redes neuronales de la corteza prefrontal" (Mora, 2021, p. 125) que consiste en la formación de mejores personas en el aula, familia, sociedad y cultura, simultáneamente. De esta manera, se sabe que el cerebro del estudiante no es software o máquina cognitiva, sino que también se debe pensar que 
es un ser humano cargado de alta dosis de afectividad. De allí se dice que en el experimento se "indicaba con claridad que el componente de comunicación, de emoción, era tan importante como pudiera serlo la misma materia impartida" (Mora, 2021, p. 195).

A partir de este experimento, que fue hace más de cuarenta años, se analiza, gracias a la transdisciplinariedad, ofrecida por el pensamiento complejo, la importancia que tiene el componente emocional en el docente y el estudiante, al momento de construir el aprendizaje. En efecto, la conexión emocional activará la vibración en el docente universitario el poder de ejercer su rol mediador, animador, guía y coach que ejerce con los estudiantes que llegan a clases, por supuesto en diversas magnitudes, con el pensamiento crítico, analítico y creativo que les motiva a "innovar, cambiar lo que conocemos y arañar pequeñas esquirlas de conocimiento nuevo" (Mora, 2021, p. 199).

Definitivamente "la emoción es la energía que mueve el mundo" (Mora, 2021, p. 69); pero no se trata de hacer postura antagónica de la educación sino de encontrar el equilibrio donde el "binomio emoción-cognición (procesos mentales) es un binomio indisoluble" (Mora, 2021, p. 71) en el ejercicio de la misión y para alcanzar la visión de una educación sustentable y sostenible por la calidad.

Por eso, la impronta emocional del docente, que forma parte de su naturaleza humana, a la hora de dar clases, le "permite generar empatía, sea con los gestos, la entonación de las palabras, la construcción de las frases y el contenido de las mismas o la facilidad de acercar y hacer entender esos contenidos al que escucha" (Mora, 2021, p. 196). Como dice el filósofo, escrito y orador Cicerón, al elogiar el valor de la palabra, el docente, al momento de impartir la clase, tiene que hablar "para emocionar, enseñar y convencer" a los estudiantes. No se trata de cuestionar la formación cognitiva que bien ha estado conduciendo la educación de la humanidad, sino que, con aporte interdisciplinar de las ciencias, se incluya y se encuentre el punto de equilibrio con la formación emocional que implica tener en cuenta diversos componentes a fin de que la calidad educativa lleve consiguientemente a una calidad de vida (Agenda 2030 y los Objetivos de Desarrollo Sostenible, objetivo 4).

\section{La impronta emocional del estudiante universitario}

Mora (2021), para hablar de la impronta emocional de los estudiantes universitarios, citó otro experimento (p. 196), realizado en 1993, por Nalini Anibady y Robert Rosenthal (Bain, 2007, p. 9). Se organizaron dos grupos de estudiantes. El primero, conformado por estudiantes que llevaron clases durante seis meses con el mismo profesor. En estos seis meses, de hecho, los estudiantes interactuaron constantemente con sus docentes a través de las clases, exposiciones, consultas, conversaciones, evaluaciones, etc. Al finalizar el curso, a los estudiantes se les entregó un cuestionario para evaluar el desempeño del docente, mediante una escala de valores, positiva y negativa.

Así mismo se designó otro grupo de estudiantes para que en corto tiempo evaluaran el desempeño del docente. Esta vez, solo se les mostró videos dando las clases con profesores a quienes nunca habían visto. A éstos, se les entregó el mismo cuestionario que se les entregó a los otros estudiantes. Los resultados fueron sorprendentes porque, en ambos grupos, se obtuvieron puntuaciones similares.

A la pregunta: ¿qué hizo que los estudiantes que escucharon la clase del profesor, a través de video y en corto tiempo, se obtuvieron resultados similares de aquellos que si interactuaron durante seis meses?

Al experimento de Mora (2021), que no dice los detalles a favor o en contra y tampoco sean necesarios mencionar, dice que "esto indica que un estudiante, ya desde el principio, recibe la impronta emocional que le permite detectar, con exquisita precisión, a un buen o a un mal profesor" (p. 196). Esa impronta emocional, manifestada por rasgos peculiares y distintivos, será pues el punto de partida de un aprendizaje fructífero que despertará la "curiosidad sagrada" (Mora, 2021, p. 191) en el estudiante que después llevará "a crear conocimiento a través de la investigación, que es la base nuclear, inviolable, del quehacer universitario" (p. 191). De allí que el cerebro del estudiante aprende solo lo que le emociona. Cabe distinguir, también, que las emociones no se reducen y tampoco se tiene que ver como una manifestación sentimental, sino que se convierte en pieza clave de un aprendizaje provechoso. Si antes se decía que la letra con sangre entra, hoy se dice que la letra con sangre no entra, sino a través de una experiencia afectiva de las emociones y efectiva de los conocimientos. En efecto, el estudiante requiere, desde la impronta emocional, experimentar la ternura del maestro que no consiste en suplir el rol paternal del hogar sino promover el amor por la educación cuyo principal objetivo es ayudar "a crecer en dignidad" (Bueno, 2021, p. 17) y desarrollar "calidad de vida" (Agenda 2030 y los Objetivos de Desarrollo Sostenible, 2015).

Se trata, pues, desde el primer momento del encuentro, de conectar emocionalmente el docente con el estudiante para rendir un aprendizaje eficiente, de corto y largo plazo, ya que "la emoción es esa energía codificada en la actividad de ciertos circuitos del cerebro que nos mantiene vivos" (Mora, 2021, p. 69). 
En este segundo experimento, descrito por Francisco Mora, los estudiantes hacen uso de su impronta emocional para darse cuenta, desde el principio, de la capacidad que tienen los docentes de lograr la conexión sináptica y la consiguiente generación de redes neuronales de aprendizaje. Es por eso que el proceso de ensamblaje cognitivo no será dependiente ni del tiempo ni del espacio, por más que existan ciertos condicionantes, sino de una experiencia motivada por el docente y desarrollada a través de "circuitos y redes neuronales de su propio cerebro" (Mora, 2021, p. 20). De allí que los resultados no diferirán, en el proceso de enseñanza-aprendizaje de la modalidad presencial en el aula con las plataformas virtuales, por más que se requiera de un proceso de alfabetización y automatización pertinentes; tampoco del tiempo que tenga que ser eficientemente en el principio, o mitad o final del semestre porque el cerebro se activa con la emoción o se desconecta con la desmotivación; de allí que existen estudiantes, no precisamente que dominan o no dominan determinados tipos de materias sino que no se despertó convenientemente la curiosidad en el estudiante (Mora, 2021, pp. 69-76), no se conectó con la atención que se pretendía (Mora, 2021, pp. 85) y por tanto no se generó aprendizaje (Mora, 2021, pp. 95-102).

No se trata de decir que el docente no pudo conectar con el estudiante sino solamente al final del semestre como si fuera un complemento, ni tampoco calificar la presencialidad como si tuviera más valor, sino que se trata de conectar con el estudiante, en todo momento, como si fuera un artista que tiene que entonar magistralmente una canción en cada concierto, dibujar una obra de arte, moldear un producto humano, diseñar un prototipo de estudiante feliz, sintiéndose plenamente un ciudadano y profesionalmente competente. Lo que si se requiere es la conciencia plena de la plasticidad cerebral "que se expresa en los cambios que son resultados sobre todo de cuanto se aprende y memoriza, de la educación que se recibe y la cultura en la que se vive" (Mora, 2021, p. 37). De allí se habla de la adaptación a las diversas circunstancias de la historia de la humanidad; como la que está viviendo estos tiempos de emergencia climática y sanitaria. Así se afrontará el reto de vivir una ciudadanía plena (PEN al 2036, 2020). Partir con el proceso envolvente de una conexión emocional hacia la conexión cognitiva en el proceso de enseñanza-aprendizaje.

Téngase en cuenta, también, que los estudiantes universitarios que están dejando las etapas difíciles de la pubertad y adolescencia pasan a una etapa diferente de su juventud, caracterizado, en el arco vital de su existencia, por actitudes de cambio de mentalidad, ansias de transformación social, proceso de maduración de conocimientos, definición de su futuro profesional, deseo de inventar soluciones a los problemas que se presentan y poseer un potencial creativo de hacer las cosas de diferente manera.

Psicológicamente están viviendo una etapa de auto aceptación por lo que se caracteriza por la sentimentalidad, reconocimiento del otro, tendencia a enamorarse. Culturalmente "es un producto, es histórico, incluye ideas, patrones y valores, es selectiva, es aprendida, está basada en símbolos y es una abstracción de la conducta y de los productos de la conducta" (Mora, 2007, p. 11); de allí que sobresalen los deseos de socialización con los amigos y sintonía con la moda globalizada del vestido, música, pensamiento, etc. que abren nuevos espacios de convivencia y diálogo "intergeneracional e interculturalmente" (PEN al 2036, p. 24). No está de menos citar, las denominaciones que les caracteriza como generación millennials que tienden, por una parte, a ser preparados y capacitados a tener varias profesiones, a la vez frágiles por el pensamiento débil y, por otra parte, inclinaciones a las tendencias youtubers, bolgers, influencers, etc. Para comprender este horizonte de cambios culturales se debe tener en cuenta “que sin ser humano como 'núcleo' no hay propiamente enseñanza ni investigación. No hay creación de pensamiento nuevo. No hay transmisión 'auténtica' de conocimientos”' (Mora, 2021, pp. 191192).

Se debe tener en cuenta, también, que sociológicamente, que los estudiantes se reúnen dentro de las aulas universitarias, con el propósito de superación y mejora en las condiciones de vida, provienen de zonas metropolitanas, urbanas, urbano-marginales, marginales, rurales, campesinas, mineras, etc.; a nivel nacional y extranjero. Cada uno de ellos con diversas condiciones étnicas, económicas, psicológicas y emocionales.

Son jóvenes que se encuentran en la cima de la salud, fuerza, energía y resistencia en el que no se trata de ser maestros que se dediquen, en el aspecto humano y profesional, a formar y replicar experiencias castrenses de uniformizar y alinear un estilos de vida, bajo el pretexto regulación de normas éticas de conducta juvenil, sino de forjar seres humanos de calidad que "enseñe a pensar y no a obedecer" (PEN al 2036, 2020, p. 81), teniendo en cuenta las influencias globalizantes de nuestros tiempos, a fin de que las universidades sean lugares "donde los estudiantes construyen, de un modo casi definitivo, los planes de su vida futura tanto profesional como personal" (Mora, 2021, p. 192). Para eso se necesita ser profesores excepcionales "que rompen las reglas, que escapan a las normas comúnmente establecidas en la enseñanza, tanto que incluso algunas veces son duramente criticados por aquellos 'puristas de la enseñanza'” (Mora, 2021, p. 194).

\section{Perfil del docente universitario}


Mientras que la Ley Universitaria, en el capítulo dedicado al docente, tiene en cuenta solo la parte cognitiva al sostener que "tiene como base fundamental la calidad intelectual y académica" (artículo 83); el Proyecto Educativo Nacional al 2036 hace una visión holística de la educación, por supuesto, considerando dentro de ello al docente, al considerar que "la cognición, el estado físico, la emoción y el desarrollo espiritual son aspectos indesligables para desarrollar todo nuestro potencial individual y colectivo" (p. 76).

Definitivamente, se debe promover en el docente el binomio indisoluble emoción-cognición para que dentro del proceso de enseñanza-aprendizaje pueda reflejar tales actitudes donde los estudiantes puedan recordar de sus docentes no "solo por su conocimiento profundo de lo que enseñaban, sino también por su comunicación empática, emocional en clase" (Mora, 2021, p. 193). Se trata de dejar huellas en el estudiante no solo por los conocimientos profundos inculcados en bien de su competitividad profesional sino también por las experiencias humanas, fraternas, paternas, solidarias, tolerantes, etc. que tiene marcadas en la impronta emocional de toda una vida. De allí que muchos profesionales recuerdan de sus docentes la manera paciente con la que fueron educados, la calidad de seres humanos que fueron en el trato y el profesionalismo con el que fueron acompañados en la madurez de sus aprendizajes.

A partir de las valoraciones hechas por los estudiantes, se describirán a continuación, las diecisiete características, basadas en estudios de la neurociencia aplicada a la educación, que debe tener un excelente y excepcional docente universitario (Bain, 2007, pp. 10-13; Mora, 2021, pp. 196-198).

1. Conocer el nivel cognitivo con el que llegan los adolescentes y jóvenes estudiantes y también la situación emocional que generará una impronta emocional en el ambiente. Así, convertirse en mediadores, acompañantes, guías y coaches en el proceso de enseñanza-aprendizaje.

2. Empático y simpático para conectar emocionalmente con el estudiante con el propósito de recorrer juntos el camino de grandes aprendizajes, lleno de sorpresas y descubrimientos.

3. Despertar la curiosidad sagrada en el estudiante, desde el inicio de clases, con el propósito de transformar lo superficial y desapercibido en algo importante y necesario; así, también, ser continuo y permanente.

4. Provocar el pensamiento crítico, analítico y creativo a través de la lectura, investigación, diálogo, debates, discusiones, desacuerdos, aclaraciones, propuestas e innovaciones.

5. Creativo en el inicio, desarrollo y cierre de las clases; utilizando anécdotas, noticias, historias, películas, canciones, memes, etc. que genere en los estudiantes el pensamiento creativo.

6. Convertir el tema en una fuente de sabiduría donde el estudiante active el deseo de querer aprender más y transformar su vida en persona y profesional excepcional, capaz de poner en práctica sus competencias y capacidades.

7. Emocionarse al momento de preparar la clase, de tal forma que el contenido se convierte en útil y necesario para la solución de los problemas del campo profesional y en la solución de los problemas de la vida.

8. Apasionado por la materia que enseña, interesado por la formación permanente y constantemente actualizado a fin de que los estudiantes tengan el pensamiento crítico, reflexivo, renovado y creativo.

9. Amante transdisciplinar de la lectura, relacionada a su materia especializada y de otras materias relacionadas; así poder interactuar y relacionarse con otras disciplinas; enriqueciendo y rindiendo aún más importante.

10. Pasión para investigar temas relacionados a su materia y publicar los frutos de su investigación personal, colegiada con otros docentes y estudiantes.

11. Compasivo por tener buen corazón y deseo de ayudar al estudiante en la consolidación de su aprendizaje. Así, realizar el proceso de enseñanza-aprendizaje a la luz de felicidad y alegría, afecto, bondad, honestidad, equidad y justicia.

12. Cultivar el arte de moldear de manera efectiva los conocimientos y de manera afectiva la personalidad del estudiante.

13. Resiliente para sobreponerse al duelo, pérdida, ausencia, crisis emocional, sentimental y temperamental ante las adversidades y dificultades que puedan suscitarse en el aula.

14. Alcanzar el éxito profesional, reflejado en los estudiantes que obtienen, a través de los procesos mentales, calidad profesional y calidad de vida, bajo el binomio emoción-cognición.

15. Sentido común que forma parte de su naturaleza humana, profesionalismo, competitividad y capacidad de poder acompañar al estudiante en su proceso emocional-cognitivo de enseñanza-aprendizaje.

16. Ser maestro, padre, amigo y confidente con el propósito de conectar con el estudiante cognitiva y emocionalmente. Así dejar huellas de calidad humana en el estudiante para toda la vida. 
17. Estilo de vida sostenible que ejerce su vocación de profesor con alegría dentro de las aulas universitarias y proyecta con esperanza un planeta saludable y seguro para el presente y las futuras generaciones.

\section{Discusión y conclusión}

Son tantos los perfiles de docentes universitarios que se han abordado actualmente, cada quien con determinada posición e interés.

Por una parte, Mas (2011) hace su investigación del perfil del profesor universitario basado en competencias haciendo "que la incorporación del docente novel sea satisfactoria, tanto para él como para la institución" (p. 208). Alfaro y Alvarado (2018) al hablar de la relación del perfil del docente universitario, sea de universidades, públicas y privadas, en la carrera de la educación dice que es importante "reconocer el perfil profesional del personal docente idóneo para implementar los planes de estudio" (p. 14). Del Águila (2019) aborda su investigación relacionada con los desempeños, entendido como el "conjunto de acciones y responsabilidades" (p. 42), en el que demuestra que si existe una relación directa y significativa. Todos ellos, expresan la necesidad de exigir el logro de una excelencia académica que definitivamente redundará en propuestas saludables que, de manera mediata e inmediata, actualizarán la calidad educativa. Ante las exigencias académicas cognitivamente remarcadas por el buen conocimiento que debe impartir el docente universitario para forjar análogamente en sus estudiantes profesionales excepcionales y excelentes, se debe tener en cuenta, en equilibrada magnitud, el "componente emocional" (Mora, 2021, p. 195) que no consiste en un paliativo sentimental sino con igual valor y significancia para la vida. Así como el ave necesita tener las dos alas en buen estado para mantener el equilibrio y poder surcar los vientos, así también el docente universitario ha de tener en cuenta el componente cognitivo y la impronta emocional en la construcción de su enseñanza-aprendizaje.

Flores (2016) que habla del perfil del docente universitario desde las dimensiones epistemológica, pedagógica, didáctica y curricular, dice que "es necesaria la definición de un perfil profesional que le permita adecuarse a la diversidad y a los continuos cambios que se operan en la sociedad del siglo XXI" (p. 114). Valerio y Rodríguez (2017) al analizar el perfil del docente universitario, desde la percepción del estudiante, en su análisis de investigación cualitativa, demuestra que la actitud del profesor, metodológica, estratégica, cognitiva y afectiva, favorece enormemente en el aprendizaje del estudiante (p.117) por lo que "resulta interesante que los factores que impactan en el aprendizaje universitario, identificados en esta investigación, coinciden en gran medida con los factores afectivos" (p. 120). Por eso, el docente universitario, además de contar con los implementos académicos y cognitivos también tiene que tener en cuenta el implemento emocional, valorizada actualmente por la neuroeducación.

Así como el ave necesita tener dos alas en buen estado, ambas en igual condiciones para elevarse libremente al viento, así también la educación necesita tener en cuenta "el binomio emoción-cognición (procesos mentales)" (Mora, 2021, p. 69) dentro del sistema educativo universitario. Se trata del equilibrio que debe considerarse dentro del perfil neuroeducativo del docente universitario excepcional y excelente; así como se tiene en cuenta en las instituciones universitarias el equilibrio entre las habilidades duras y habilidades blandas. En la medida que se tenga tales consideraciones cognitivas y emocionales habrá bienestar emocional en el docente, calidad educativa en la universidad, impacto cualitativo en el estudiante y la construcción de un mundo mejor. Gracias a la impronta emocional que dota de rasgos peculiares y distintivos.

De allí que esta investigación propone, a modo de conclusión, diecisiete características, remarcadas y valoradas por la impronta emocional, como rasgos peculiares y distintivos del docente universitario, en el perfil del docente universitario que corroboran en el cumplimiento de las condiciones básicas de la calidad en la investigación, gestión académica administrativa y responsabilidad social universitaria.

\section{Referencias}

Alfaro Rojas , G., \& Alvarado Cordero , S. (01 de Mayo de 2018). El Perfil de profesores universitarios de universidades públicas y privadas en la carreraa de Educación. Revista Electrónica "Actualidades Investigativas en Educación", 18(2), 1-21: https://doi.org/10.15517/aie.v18i2.33161

Bain, K. (2007). Lo que hacen los mejores profesores de la universidad. (U. d. Valencia, Ed.) https://www.academia.edu/8601238/LO_QUE_HACEN_LOS_MEJORES_PROFESORES_UNIVERSITARIO S_Libro_completo_ 
Bueno i Torrens, D. (2021). Neurociencia para educadores: Todo lo que los educadores siempre han querido saber sobre el cerebro de sus alumnos y nunca nadie se ha atrevido a explicárselo de manera comprensible y útil (Sexta ed.). Octaedro - Rosa Sensat.

Del Águila Riva, R. (2019). Pefil Profesional del Profesor Universitario y su Relación con el Desempeño Docente. Perú: Universidad San Martí de Porres. https://repositorio.usmp.edu.pe/handle/20.500.12727/6073

Flores Correa , L. (2016). Perfil docente del profesorado UDEP y su relación con e Idesarrolo de las funciones docentes universitarias. Universidad de Piura. https://pirhua.udep.edu.pe/bitstream/handle/11042/2755/MAE_EDUC_335.pdf?sequence=1\&isAllo wed=y

Fuentes , A., \& Collado, J. (2019). Fundamentos epistemológicos transdisciplinares de educación y neurociencia. Sophía: Colección de la educación, 26(1), 83-113.

Gobierno del Perú. (2014). Ley Universitaria 30220. Ministerio de Educación. http://www.minedu.gob.pe/reforma-universitaria/pdf/ley_universitaria.pdf

Mas Torelló, O. (04 de Diciembre de 2011). El profesor universitario: sus competencias y formación. Revista de Currículum y formación del Profesorado, 15(3), 195-211. https://www.ugr.es/ recfpro/rev153COL1.pdf

Mora Teruel, F. (2007). Neurocultura: una cultura basada en el cerebro. Alianza Editorial. https://drive.google.com/file/d/1VA9olagkStwzGmnnPYMuJo9r-0hfiYJO/view?fbclid=IwAR2n5iYEQI2rELrUUZp3E9Z6gIdNp8KJvSBMIxukBdAMr7rEuUcfk1nK_4

Mora Teruel, F. (2021). Neuroeducación: solo se puede aprender aquello que se ama (Tercera ed.). Alianza Editorial .

Moreno, J., \& Marcaccio, A. (2014). Prefiles Profesionales y Valores Relativos al trabajo. Ciencias Psicológicas, VIII(2), 129-138. http://www.scielo.edu.uy/pdf/cp/v8n2/v8n2a03.pdf

Morin, E. (2011). Introducción al pensamiento complejo. España : Gedisa Editorial . http://cursoenlineasincostoedgarmorin.org/images/descargables/Morin_Introduccion_al_pensamien to_complejo.pdf

Ocampo, J., Castro, W., Becerra , G., \& Herrera, B. (2014). Caracterización del Perfil del docente en los Programas de Administración y sus competencias para la docencia. Colombia: Asociación Colombiana de Facultades de Administración, Ascolfa. https://www.ascolfa.edu.co/wcontent/uploads/2015/07/LIBRO_CARACTERIZACION_DEL_PERFIL_DEL _DOCENTE_DE_LOS_PROGRAMAS_DE_ADMINISTRACION.pdf

Organización de las Naciones Unidas . (2018). La Agenda 2030 y los Objetivos de Desarrollo Sostenible: Una oportunidad para América Latina y El Caribe. Comisión Económica para América Latina y El Caribe (CEPAL). https://www.cepal.org/es/publicaciones/40155-la-agenda-2030-objetivos-desarrollosostenible-oportunidad-america-latina-caribe

Oviedo, Y. (2009). Competencias docentes para enfrentar la sociedad del conocimiento. Apertura Revista de Innovación Educativa,

1(1). http://www.udgvirtual.udg.mx/apertura/index.php/apertura/article/view/117/118

Valerio Ureña, G., \& Rodríguez Martínez, M. (Mayo-agosto de 2017). Perfil del profesor universitario desde la perspectiva del estudiante. Innovación Educativa, 17(74), 109-124. http://www.scielo.org.mx/pdf/ie/v17n74/1665-2673-ie-17-74-00109.pdf 